\title{
Zinc transporter 8 autoantibody in the diagnosis of type 1 diabetes in children
}

Nur Rochmah, MD, Muhammad Faizi, MD, Siti Wahyu Windarti, MD

Department of Child Health, Dr. Soetomo General Hospital, Faculty of Medicine, Universitas Airlangga, Surabaya, Indonesia

Background: Type 1 diabetes (T1D) is an organ-specific autoimmune disease related to the autoimmune response against pancreatic $\beta$-cells. Zinc transporter 8 (ZnT8), an islet-specific gene product localized to the $\beta$-cell insulin granule, was recently identified as an autoantigen in T1D.

Purpose: To evaluate the use of $\mathrm{ZnT} 8$ autoantibody (ZnT8A) for diagnosing T1D.

Methods: This case-control study was conducted at Dr. Soetomo General Hospital, Surabaya, Indonesia, from March to May 2019. Children younger than 18 years of age with T1D based on the International Society for Pediatric and Adolescent Diabetes guideline and healthy controls were included. We measured ZnT8A level using enzyme-linked immunosorbent assay (cutoff value, 0.315 ).

Results: There were 30 children with T1D (50.0\% boys; mean age, $11.3 \pm 3.7$ years) and 18 healthy controls $(44.4 \%$ boys; mean age, $8.3 \pm 3.1$ years); 1 patient in each group was Madurese, while the others were Javanese. Twenty-two of 30 subjects with T1D (73.3\%) tested positive for ZnT8A com pared to 5 of 18 controls $(27.8 \%)(P=0.02$; odds ratio, 7.15; 95\% confidence interval, 1.93-26.52). When ZnT8A-positive and -negative T1D cases were compared, no differences were detected in age at diagnosis, duration of diabetes, presence of ketoacidosis, body mass index, glycosylated hemoglobin concentration, or C-peptide concentrations.

Conclusion: ZnT8A may be useful in the diagnosis of T1D.

Key words: ZnT8 autoantibody, Type 1 diabetes, Diagnosis, Child

\section{Key message}

Question: Can zinc transporter 8 autoantibody (ZnT8A) be used for diagnosing type 1 diabetes (T1D)?

Finding: Twenty-two of 30 subjects with type 1 diabetes $(73.3$ \%) were positive for ZnT8A compared to 5 of 18 controls (27.8\%).

Meaning: ZnT8A has potential for clinical applications in the diagnosis of T1D.

\section{Introduction}

Type 1 diabetes (T1D) is a kind of organ-specific autoimmune disease related to the autoimmune response against pancreatic $\beta$-cells. Measurement of the autoantibodies against pancreatic $\beta$-cell antigens, such as glutamic acid decarboxylase antibody (GADA), anti-insulin autoantibody (IAA), insulinoma-associated antigen-2 antibody (IA-2A), and islet cell autoantibody, is helpful to diagnose T1D. Zinc transporter 8 (ZnT8), an islet-specific gene product localized to the $\beta$-cell insulin granule, is recently identified as an autoantigen in T1D. ${ }^{1)}$ The introduction of zinc transporter 8 autoantibody (ZnT8A) in the routine diagnostic process of T1D may improve the overall autoantibody sensitivity. ${ }^{2)}$ Positivity of ZnT8A in T1D is related to the early onset of illness and increases the risk of diabetic ketoacidosis (DKA) as one ofthe complications. ${ }^{3)}$ The presence of ZnT8A in T1D was associated with ethnic that could be explained by genetic factors. ${ }^{4,5)}$ To date, study about ZnT8A as a diagnostic parameter of T1D is still limited in Asia Pacific region, especially in Indonesia. Therefore, we aimed to evaluate the use of ZnT8A for diagnosing T1D.

\section{Methods}

This case-control study was approved by the ethical com. mittees of the Dr. Soetomo General Hospital, Surabaya, Indonesia (1131/KEPK/IV/2019). Written informed consent was obtained from all participants. This study was conducted in the outpatient clinic of Endocrinology Department of Dr. Soetomo General Hospital, Surabaya, Indonesia from March to May 2019. A total of 30 patients, between $1-18$ years of age (15 boys and 15 girls) diagnosed with T1D according to International Society for Pediatric and Adolescent Diabetes guideline, ${ }^{6}$ were included. Eighteen healthy children with no diabetes or other autoimmune diseases were included in the study as controls.

We interviewed the subjects to collect data on their demographic, height, and weight. Body mass index (BMI, $\left.\mathrm{kg} / \mathrm{m}^{2}\right) z$ score

\footnotetext{
Corresponding author: Nur Rochmah, MD. Department of Child Health, Dr. Soetomo General Hospital / Faculty of Medicine, UniversitasAirlangga, Address: Mayjend Prof. Dr. Moestopo No. 6-8, Surabaya, East Java 60286, Indonesia 凶 E-mail: drnurrochmah@gmail.com, https://orcid.org/0000-0002-9626-9615

Received: 1 October, 2019, Revised: 11 March, 2020, Accepted: 28 March, 2020

This is an open-access article distributed under the terms of the Creative Commons Attribution Non-Commercial License (http://creativecommons.org/licenses/by$\mathrm{nc} / 4.0 /$ ) which permits unrestricted non-commercial use, distribution, and reproduction in any medium, provided the original work is properly cited.

Copyright (c) 2020 by The Korean Pediatric Society
} 


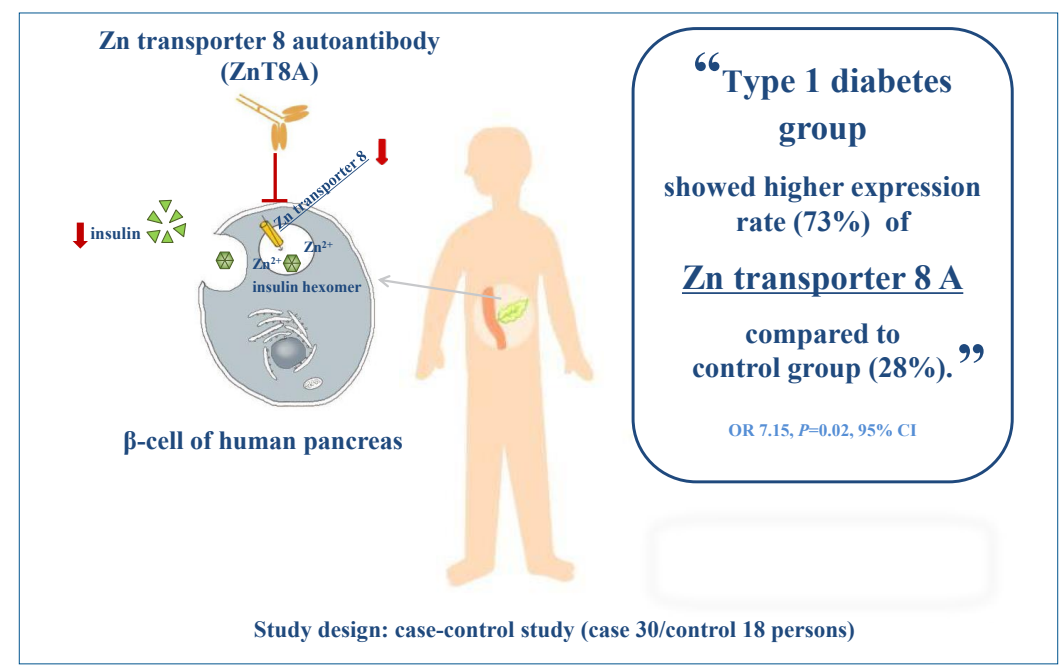

Graphical abstract. Total population (children with type 1 diabetes mellitus, T1DM) at Department of Child Health of General Hospital Dr. Soetomo were 60. Thirty children with T1DM were included into diabetes group and 18 healthy children were included into control group. We examined zinc transporter 8 autoantibody (ZnT8A) using enzyme-linked immunosorbent assay kit. We found that positivity ZnT8A in 22/30 children with T1DM (73.3\%) and 5/18 in healthy children (27.8\%). We conclude that diabetes group showed higher expression of ZnT8A compared to control group, so ZnT8A has potential for clinical application in the diagnosis of T1DM.

Table 1. Patients' baseline characteristics

\begin{tabular}{|c|c|c|c|}
\hline Characteristic & T1D $(n=30)$ & Controls $(n=18)$ & $P$ value \\
\hline \multicolumn{4}{|l|}{ Sex } \\
\hline Male & $15(50.0)$ & $8(44.4)$ & \\
\hline Female & $15(50.0)$ & $10(55.6)$ & \\
\hline Age (yr) & $11.3 \pm 3.7$ & $8.3 \pm 3.1$ & \\
\hline Weight (kg) & $27.0(1365)$ & $27.0(1157)$ & \\
\hline Height (cm) & $132.1 \pm 19.3$ & $124.2 \pm 18.5$ & \\
\hline Body mass index $\left(\mathrm{kg} / \mathrm{m}^{2}\right)$ & $16.7 \pm 4.6$ & $19.5 \pm 5.2$ & \\
\hline \multicolumn{4}{|l|}{ Ethnicity } \\
\hline Javanese & $29(96.7)$ & $17(94.4)$ & \\
\hline Madurese & $1(3.3)$ & $1(5.6)$ & \\
\hline C-peptide level (ng/mL) & $0.01(0.01-2.49)$ & $1.28(0.29-3.83)$ & $<0.001^{a)}$ \\
\hline
\end{tabular}

Values are presented as number (\%), mean \pm standard deviation, median (range).

a) Mann-Whitney $U$ test

Boldface indicates a statistically significant difference with $P<0.05$.

was computed. We collected the data about age at diagnosis, duration of diabetes, and history of DKA. Ketosis was diagnosed by the elevation of serum ketone bodies. ${ }^{7}$ ZnT8A level was analyzed using enzyme-linked immunosorbent assay (ELISA) kit (Bioassay Technology Laboratory, Birmingham, UK) with the positive of cutoff value was $0.315 .^{8}$

Data were analyzed using SPSS ver. 17.0 (SPSS Inc., Chicago, IL, USA). After testing for normality with Kolmogorov-Smirnov test, all normally distributed data were expressed as mean \pm standard deviation, and nonnormally distributed data were expressed as the median (interquartile range). Categorical data were expressed with numbers or percentage. Frequency differences were compared with chi-square test or Fisher exact test when appropriate. Differences in parametric data were tested
Table 2. Clinical characteristics of patients with type 1 diabetes

\begin{tabular}{lccc}
\hline Variable & $\begin{array}{c}\text { ZnT8A-positive } \\
(\mathrm{n}=22)\end{array}$ & $\begin{array}{c}\text { ZnT8A-negative } \\
(\mathrm{n}=8)\end{array}$ & $P$ value \\
\hline Age at diagnosis (yr) & $6.8 \pm 3.6$ & $7.9 \pm 4.0$ & $\left.0.483^{\mathrm{a}}\right)$ \\
Duration of diabetes $(\mathrm{yr})$ & $4.1 \pm 3.0$ & $4.6 \pm 3.2$ & $\left.0.686^{\mathrm{a}}\right)$ \\
Ketosis $(\%)$ & 86.4 & 87.5 & $0.716^{\mathrm{b}}$ \\
Body mass index $\left(\mathrm{kg} / \mathrm{m}^{2}\right)$ & $14.3(10.8-30)$ & $16.0(15.1-22.3)$ & $0.241^{\mathrm{c}}$ \\
HbA1c (\%) & $8.5(6-21)$ & $7.0(7-11)$ & $0.285^{\mathrm{c}}$ \\
C-peptide level $(\mathrm{ng} / \mathrm{mL})$ & $0.05(0.01-2.49)$ & $0.01(0.01-0.78)$ & $0.161^{\mathrm{c}}$ \\
\hline
\end{tabular}

Values are presented as mean \pm standard deviation and median (range). $\mathrm{HbA1C}$, glycosylated hemoglobin.

${ }^{a)}$ Independent $t$ test. ${ }^{\text {b) }}$ Fisher exact test. ${ }^{c}$ Mann-Whitney $U$ test.

by Independent $t$ test. Differences in nonparametric data were analyzed by the Mann-Whitney $U$ test. $P$ value less than 0.05 were considered statistically significant.

\section{Results}

There were 30 children with T1D (15 boys and 15 girls; aged $11.3 \pm 3.7$ years) and 18 healthy children ( 8 boys and 10 girls; aged $8.3 \pm 3.1$ years) included in this case-control study. The baseline characteristics of the patients with T1D and the controls were presented in Table 1 . Twenty-two out of 30 subjects $(73.3 \%)$ with T1D were positive for ZnT8A compared to 5 of 18 controls (27.8\%) $(P=0.02$; odds ratio, 7.15 ; 95\% CI, 1.93-26.52). The history-based, anthropometric, and metabolic characteristics of the patient with T1D were shown in Table 2. When ZnT8Apositive and -negative in T1D cases were compared, no difference was detected in age at diagnosis, duration of diabetes, presence of ketoacidosis, BMI $z$ score, glycosylated hemoglobin 


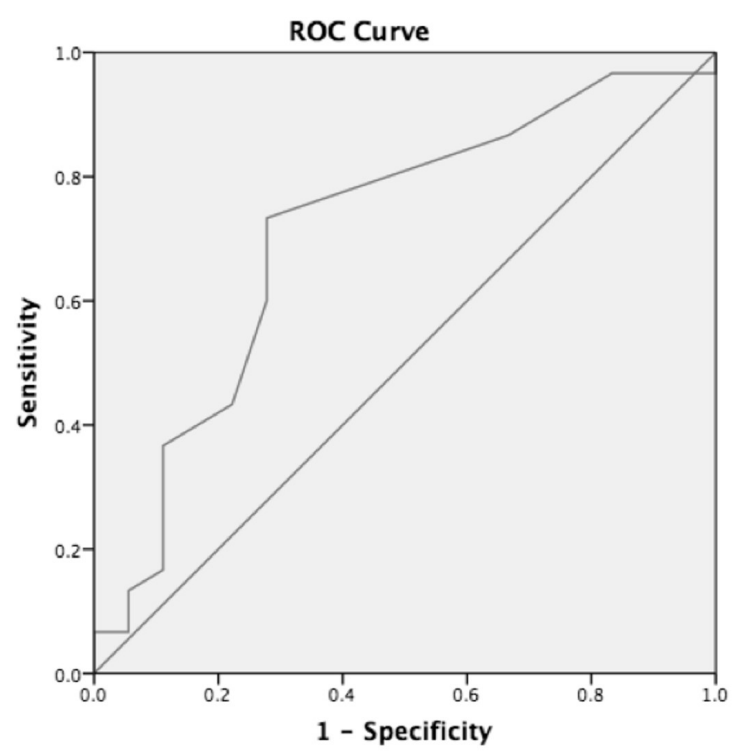

Fig. 1. Receiver operating characteristic (ROC) curve for Zinc transporter 8 as a screening test for type 1 diabetes mellitus.

concentration, and C-peptide concentrations. These variables were examined during the study.

An area under the curve (AUC) of 0.710 (95\% CI, 0.5530.867). According to ROC analysis, ZnT8A demonstrated sensitivity $73.3 \%$ and specificity $72.2 \%$ for diagnosing T1D at cutoff of 0.315 (Fig. 1).

\section{Discussion}

This study showed that ZnT8A-positive is $73.3 \%$ in Javanese children with T1D. This result is in accordance with most of the studies done in other countries. ZnT8A positivity was reported to be between $60 \%-80 \%$ in Caucasians (11-8 years old), $72 \%$ in Czechs (1-19 years old), 65\% in Argentinians (10-32 years old), and $58.6 \%$ in Turkish children (1-18 years old) with new onset T1D. ${ }^{9-12)}$ ZnT8A positivity was reported in $24 \%$ of Chinese new onset T1D patients (1-70 years old) and differences in human leukocyte antigen (HLA) genotypes or other interethnic genetic markers were thought to be a possible cause for this low rate. ${ }^{13)}$ However, in another Asian population, Japanese acute onset T1D patients $(19.1 \pm 14.5$ years old) had $58 \%$ ZnT8A positivity. ${ }^{14)}$ A study from Brazil, which encompassed both a Caucasian and a non-Caucasian new onset T1D population (30.3 \pm 11.4 years old), found an overall ZnT8A positivity of $24 \%$ and it was stated that neither ZnT8A positivity nor concentration was associated with ethnicity. ${ }^{15)}$ The differences in prevalence of various types of islet autoantibodies among different ethnic populations with T1D could be explained by genetic factors especially HLA genotypes and SLC30A8 gene polymorphism. ${ }^{4,5)}$ Additionally, a recent international prospective cohort study identified a number of non-HLA genes as genetic factors for development of islet autoimmunity and T1D. ${ }^{16)}$

The ZnT8A positivity prevalence of healthy controls from different countries was reported as $1 \%-2.7 \%$, which is a markedly lower rate than in this study $(27.8 \%) .2,8,9,13)$ This difference may be attributed to the larger cohorts of the other studies which better reflects the population. However, the possible effect of ethnicity cannot be excluded. ZnT8A was shown to predict risk of progression to T1D in first degree relatives of T1D patients. ${ }^{17)}$ Although these healthy controls had a negative T1D history in their families, they may have a higher risk for diabetes.

Yang et al. ${ }^{13)}$ found no difference in ZnT8A prevalence in subjects stratified with duration of diabetes. Collectively, there was no difference between the duration of type 1 diabetic patients with ZnT8A-positive and ZnT8A-negative in the present study. Another study reported that $61 \%$ of patients with T1D onset were positive for ZnT8A, and the proportion was only $33 \%$ in patients with T1D for more than 6 months. ${ }^{18)}$ However, Howson et al. ${ }^{19)}$ demonstrated a higher prevalence of ZnT8A in the first 2 years of the disease. The factors involved in the maintenance of serum ZnT8A are still to be identified, but this characteristic makes the measurement of this antibody particularly useful in patients with long-standing diabetes and unclear diabetes classification, where the identification of an autoimmune marker can establish the definite diagnosis. ${ }^{20)}$

In this study, we found the ZnT8A-positive in the T1D patient had younger age at diagnosis than with ZnT8A-negative but not significantly difference. Vaziri-Sani et al. ${ }^{21)}$ found ZnT8A titers were significantly higher in the 2- to 17-year-old type 1 diabetic patients as compared to the 15- to 34-year-old. Nevertheless, another study, which was conducted in children and adolescents who were younger than 15 years and were newly diagnosed with diabetes, reported that the ZnT8A positivity was associated with older age. ${ }^{3)}$ As above, varying ages may lead to different effects on the ZnT8A frequency. Likewise, age of onset also has an effect on the ZnT8A frequency. It was reported that the prevalence of ZnT8A declined with the increasing age at diagnosis in Chinese and Belgian patients with T1D. ${ }^{13,22)}$ Besides, the children aged 6-10 years had higher prevalence of ZnT8A than other older French within 6 months of T1D onset. ${ }^{18)}$ Taken together, it is speculated that the age at diagnosis may play an important role in the $\mathrm{ZnT}$ 8A level.

Mbanya et al. ${ }^{23)}$ showed that the ZnT8A-positive patients with diabetes had significantly lower BMI $z$ scores than the ZnT8Anegative ones. In the present study, the ZnT8A-positive type 1 diabetics had lower BMI $z$ scores than the ZnT8A-negative type 1 diabetics, but not significantly.

The Diabetes Antibody Standardization Program workshop identified that ZnT8A measured by radioligand assay showed a median ROC-AUC of $0.848 .{ }^{24)}$ Dunseath et al. ${ }^{25)}$ showed an AUC of $0.80,54 \%$ sensitivity, and $99 \%$ specificity by conducting ROC analysis of ZnT8A by ELISA in type 1 diabetics and healthy blood donors. In this study, the ROC-AUC for ZnT8A by ELISA is $0.71,73.3 \%$ sensitivity, and $72.2 \%$ specificity. Hence, we speculate that the ZnT8A test by ELISA may have high accuracy, not lower than the test by radioligand assay.

ZnT8A was recently identified as an autoantibody marker in 
T1D, which is detected earlier rather than other autoantibodies (IAA, IA-2A, and GADA) and increase diagnostic sensitivity of T1D. ${ }^{26}$ The study is limited by a relatively small number of subjects. The conclusion of this study is ZnT8A has potential for clinical applications in the diagnosis of T1D.

\section{Conflicts of interest}

No potential conflict of interest relevant to this article was reported.

\section{Acknowledgments}

The authors wish to thank the patients who participated in the study and the endocrine teams of Dr. Soetomo Hospital, Surabaya, Indonesia, for the support.

\section{References}

1. Wenzlau JM, Frisch LM, Gardner TJ, Sarkar S, Hutton JC, Davidson HW. Novel antigens in type 1 diabetes: the importance of ZnT8. Curr Diab Rep 2009;9:105-12.

2. Fabris M, Zago S, Liguori M, Trevisan MT, Zanatta M, Comici A, et al. Anti-zinc transporter protein 8 autoantibodies significantly improve the diagnostic approach to type 1 diabetes: an Italian multicentre study on paediatric patients. Auto Immun Highlights 2015;6:17-22.

3. Juusola M, Parkkola A, Härkönen T, Siljander H, Ilonen J, Åkerblom $\mathrm{HK}$, et al. Positivity for zinc transporter 8 autoantibodies at diagnosis is subsequently associated with reduced $\beta$-cell function and higher exogenous insulin requirement in children and adolescents with type 1 diabetes. Diabetes Care 2016;39:118-21.

4. Delli AJ, Vaziri-Sani F, Lindblad B, Elding-Larsson H, Carlsson A, Forsander $\mathrm{G}$, et al. Zinc transporter 8 autoantibodies and their association with SLC30A8 and HLA-DQ genes differ between immigrant and Swedish patients with newly diagnosed type 1 diabetes in the Better Diabetes Diagnosis study. Diabetes 2012;61:2556-64.

5. Wenzlau JM, Liu Y, Yu L, Moua O, Fowler KT, Rangasamy S, et al. A common nonsynonymous single nucleotide polymorphism in the SLC30A8 gene determines ZnT8 autoantibody specificity in type 1 diabetes. Diabetes 2008;57:2693-7.

6. Craig ME, Jefferies C, Dabelea D, Balde N, Seth A, Donaghue KC, et al. ISPAD Clinical Practice Consensus Guidelines 2014. Definition, epidemiology, and classification of diabetes in children and adolescents. Pediatr Diabetes 2014;15 Suppl 20:4-17.

7. Kawasaki E, Maruyama T, Imagawa A, Awata T, Ikegami H, Uchigata Y, et al. Diagnostic criteria for acute-onset type 1 diabetes mellitus (2012): Report of the Committee of Japan Diabetes Society on the Research of Fulminant and Acute-onset Type 1 Diabetes Mellitus. J Diabetes Investig 2014;5:115-8.

8. Unal I. Defining an optimal cut-point value in ROC analysis: an alternative approach. Comput Math Methods Med 2017;2017:3762651.

9. Wenzlau JM, Juhl K, Yu L, Moua O, Sarkar SA, Gottlieb P, et al. The cation efflux transporter ZnT8 (Slc30A8) is a major autoantigen in human type 1 diabetes. Proc Natl Acad Sci US A 2007;104:17040-5.

10. Petruzelkova L, Ananieva-Jordanova R, Vcelakova J, Vesely Z, Stechova $\mathrm{K}$, Lebl J, et al. The dynamic changes of zinc transporter 8 autoantibodies in Czech children from the onset of Type 1 diabetes mellitus. Diabet Med 2014;31:165-71.

11. Faccinetti NI, Guerra LL, Penas Steinhardt A, Iacono RF, Frechtel GD, Trifone L, et al. Characterization of zinc transporter 8 (ZnT8) antibodies in autoimmune diabetic patients from Argentinian population using monomeric, homodimeric, and heterodimeric $\mathrm{ZnT} 8$ antigen variants.
Eur J Endocrinol 2016;174:157-65.

12. Elmaoğulları S, Uçaktürk SA, Elbeg Ş, Döğer E, Tayfun M, Gürbüz F, et al. Prevalence of ZnT8 antibody in turkish children and adolescents with new onset type 1 diabetes. J Clin Res Pediatr Endocrinol 2018;10:10812.

13. Yang L, Luo S, Huang G, Peng J, Li X, Yan X, et al. The diagnostic value of zinc transporter 8 autoantibody (ZnT8A) for type 1 diabetes in Chinese. Diabetes Metab Res Rev 2010;26:579-84.

14. Kawasaki E, Nakamura K, Kuriya G, Satoh T, Kobayashi M, Kuwahara $\mathrm{H}$, et al. Zinc transporter 8 autoantibodies in fulminant, acute-onset, and slow-onset patients with type 1 diabetes. Diabetes Metab Res Rev 2011;27:895-8.

15. Araujo DB, Skärstrand H, Barone B, Dantas JR, Kupfer R, Zajdenverg L, et al. Zinc transporter 8 autoantibodies in patients with type 1 diabetes from a multiethnic population and their first degree relatives. Arq Bras Endocrinol Metabol 2014;58:737-43.

16. Sharma A, Liu X, Hadley D, Hagopian W, Chen WM, Onengut-Gumuscu $\mathrm{S}$, et al. Identification of non-HLA genes associated with development of islet autoimmunity and type 1 diabetes in the prospective TEDDY cohort. J Autoimmun 2018;89:90-100.

17. Gorus FK, Balti EV, Vermeulen I, Demeester S, Van Dalem A, Costa O, et al. Screening for insulinoma antigen 2 and zinc transporter 8 autoantibodies: a cost-effective and age-independent strategy to identify rapid progressors to clinical onset among relatives of type 1 diabetic patients. Clin Exp Immunol 2013;171:82-90.

18. Garnier L, Marchand L, Benoit M, Nicolino M, Bendelac N, Wright C, et al. Screening of ZnT8 autoantibodies in the diagnosis of autoimmune diabetes in a large French cohort. Clin Chim Acta 2018;478:162-5.

19. Howson JM, Krause S, Stevens H, Smyth DJ, Wenzlau JM, Bonifacio E, et al. Genetic association of zinc transporter 8 (ZnT8) autoantibodies in type 1 diabetes cases. Diabetologia 2012;55:1978-84.

20. Trabucchi A, Faccinetti NI, Guerra LL, Puchulu FM, Frechtel GD, Poskus E, et al. Detection and characterization of ZnT8 autoantibodies could help to screen latent autoimmune diabetes in adult-onset patients with type 2 phenotype. Autoimmunity 2012;45:137-42.

21. Vaziri-Sani F, Oak S, Radtke J, Lernmark K, Lynch K, Agardh CD, et al. ZnT8 autoantibody titers in type 1 diabetes patients decline rapidly after clinical onset. Autoimmunity 2010;43:598-606.

22. Vermeulen I, Weets I, Asanghanwa M, Ruige J, Van Gaal L, Mathieu C, et al. Contribution of antibodies against IA- $2 \beta$ and zinc transporter 8 to classification of diabetes diagnosed under 40 years of age. Diabetes Care 2011;34:1760-5.

23. Mbanya A, Ngandeu A, Kamwa V, Donfack OT, Lontchi É, Leke R, et al. Metabolic features associated with positivity to ZnT8 autoantibody in sub-Saharan African young-onset diabetes patients. Diabetes Metab 2016;42:204-6.

24. Lampasona V, Schlosser M, Mueller PW, Williams AJ, Wenzlau JM, Hutton JC, et al. Diabetes antibody standardization program: first proficiency evaluation of assays for autoantibodies to zinc transporter 8. Clin Chem 2011;57:1693-702.

25. Dunseath G, Ananieva-Jordanova R, Coles R, Powell M, Amoroso M, Furmaniak J, et al. Bridging-type enzyme-linked immunoassay for zinc transporter 8 autoantibody measurements in adult patients with diabetes mellitus. Clin Chim Acta 2015;447:90-5.

26. Yi B, Huang G, Zhou ZG. Current and future clinical applications of zinc transporter-8 in type 1 diabetes mellitus. Chin Med J (Engl) 2015; 128:2387-94.

How to cite this article: Rochmah N, Faizi M, Windarti SW. Zinc transporter 8 autoantibody in the diagnosis of type $1 \mathrm{di}$ abetes in children. Clin Exp Pediatr 2020;63:402-5. https://doi. org/10.3345/cep.2019.01221 